\title{
Waves in asymmetric hyperbolic media
}

\author{
S. M. Hashemi, ${ }^{* 1,2}$ I. S. Nefedov, ${ }^{1}$ and M. Soleimani ${ }^{2}$ \\ ${ }^{1}$ Aalto University, School of Electrical Engineering, SMARAD Center of Excellence, P.O. Box 13000, 00076 Aalto, \\ Finland, \\ ${ }^{2}$ Iran University of Science and Technology, Department of Electrical Engineering, Tehran 1684613114, Iran.
}

Received June 05, 2013; accepted June 28, 2013; published June 30, 2013

\begin{abstract}
In this paper, a homogenization model is applied for analysis of the spectrum of natural modes in s finite-thickness slab of tilted metallic carbon nanotubes. Tilted anisotropy axis causes a difference between normal wave vector components for waves, propagating upward and downward with respect to slab interfaces. This asymmetry effect becomes very strong for hyperbolic media and results in the appearance of backward waves and accumulation points in the spectra of natural waves.
\end{abstract}

Nowadays there is a rapidly growing interest and number of publications devoted to hyperbolic media (HM), i.e. media possessing hyperbolic-type dispersion in the space of wave vectors. Hyperbolic dispersion is inherent in uniaxial materials in which the axial and tangential components of a permittivity tensor have different signs [1], for the TEM waves in wire media [2], arrays of metallic carbon nanotubes [3]. Hyperbolic media (HM) exhibit such remarkable properties as a capability to support propagating waves with very large components of a wave vector and a very high electromagnetic density of states, which results in their potential applications in photonics because all physical processes associated with spontaneous emission become strongly enhanced [4,5]. Hyperbolic dispersion may cause the propagation of backward waves in magnetized ferrite films [6], in wiremedium slabs [7] and finite-thickness slabs of vertically standing carbon nanotubes [8]. A strong and even giant enhancement of near-field thermal radiative heat transfer between two bodies in the presence of HM [9-13] seems to be promising for the creation of new types of thermophotovoltaic systems.

In this paper we discuss the dispersion properties of waves propagating in slabs of single-wall metallic carbon nanotubes (CNT), tilted with respect to slab interfaces, see the schematic view in Fig. 1. We assume, for simplicity, that the CNT slab is grounded by the perfect electric conductor plane at $\mathrm{z}=0$. Losses are neglected. A graphical method of determining the TM wave numbers of the surface wave modes is introduced, which clearly identifies different conditions of propagation, depending on the properties of the CNT slab. In the framework of an effective medium theory, the arrays of CNTs, infinitely long in the $z^{\prime}$ direction and periodic in the $x^{\prime}$ and $y^{\prime}$ directions, can be described in terms of a permittivity dyadic

$$
\overline{\bar{\varepsilon}}^{\prime}=\varepsilon_{z z}^{\prime} z_{0}^{\prime} z_{0}^{\prime}+\varepsilon_{t}\left(x_{0}^{\prime} x_{0}^{\prime}+y_{0}^{\prime} y_{0}^{\prime}\right)
$$

where $\varepsilon_{t}$ is the transversal component of the permittivity dyadic and $\varepsilon_{z z}^{\prime}$ can is found using the effective medium model for arrays of metallic CNTs [8]. The quantum properties of individual CNTs are taken into account via the model of dynamic surface conductivity and effective boundary conditions [14]. Then, rotating by the angle $\phi$ around the $y^{\prime}$ axis, the permittivity dyadic is transformed to the coordinate system $X O Z$, associated with slab interfaces. The expression for the normal components $k_{z}$ of the wave vector is found from Maxwell equations and reads as [15]

$$
k_{z}^{(1,2)}=\frac{k_{x} \varepsilon_{x z} \pm \sqrt{\Delta k_{c}^{2}}}{\varepsilon_{z z}},
$$

where $\Delta=\varepsilon_{x x} \varepsilon_{z z}-\varepsilon_{x z}{ }^{2}$ and $k_{c}^{2}=k_{0}^{2} \varepsilon_{z z}-k_{x}^{2}$. It is remarkable that $k_{z}$ components can be strongly different for waves propagating upward and downward with respect to interfaces under fixed $k_{x}$. By this reason, we term the media possessing such properties asymmetric hyperbolic media (AHM). Another value needed for any interface solution is the transverse wave impedance $Z_{1,2}$, which for the TM waves reads as [15]:

$$
Z_{1}=-Z_{2}=\frac{-E_{x}}{H_{y}}=\frac{\eta}{k_{0}} \frac{\sqrt{k_{0}^{2} \varepsilon_{z z}-k_{x}^{2}}}{\sqrt{\varepsilon_{x x} \varepsilon_{z z}-\varepsilon_{x z}^{2}}}=\frac{\eta}{k_{0}} \frac{k_{c}}{\sqrt{\Delta}},
$$

where $\eta=120 \pi \Omega$. Then, using the $2 \times 2$ transfer matrix $[\mathrm{M}]$, suitable for asymmetric and non-reciprocal media (see [15]), we obtain the dispersion equation in the form:

\footnotetext{
*E-mail: seyedmohammad.hashemi@aalto.fi
} 


$$
M_{22}=\frac{1}{Z_{0}} M_{12} .
$$

Formula (4) can be simplified to:

$$
j Z_{0}=Z_{1} \tan \left(k_{z}^{\prime} h\right)
$$

where $Z_{0}$ and $k_{z}^{\prime}$ are defined respectively by:

$$
\begin{gathered}
Z_{0}=\frac{\eta}{k_{0}} k_{z 0}, \quad k_{z 0}=\sqrt{k_{0}^{2}-k_{x}^{2}} \\
k_{z}^{\prime}=\frac{\sqrt{\Delta \times k_{c}^{2}}}{\varepsilon_{z z}}
\end{gathered}
$$

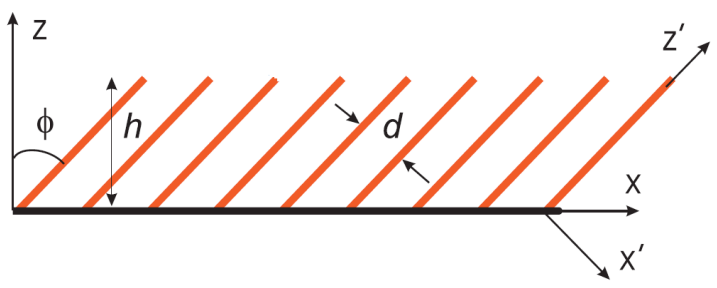

Fig. 1. Schematic view of an indefinite-medium slab with a tilted optical axis

We consider a grounded CNT indefinite medium slab of thickness $h=0.3 \lambda_{0} \quad\left(f_{0}=20 \mathrm{THz}\right)$ in free space [9]. The diagonal components of the permittivity dyadic $\varepsilon_{x x}$ and $\varepsilon_{z z}$ become zero at the frequency $f_{0}$. The grounded slab of HM may support surface-wave modes with respect to the direction of propagation ( $x$-direction). The TM modes (with the electric field perpendicular to the ground) interact with the CNT when propagating as in the grounded slab. Here we present a graphical solution of the dispersion Eq. (5) involved to determine the values of the TM wave numbers. In using this graphical technique, we also determine the number of possible modes and whether a particular mode has a cutoff or not.

In particular, surface waves have a purely imaginary transverse wavenumber in free space with $k_{z 0}=-\mathrm{j} \alpha_{z 0}$ for proper surface waves $\left(\alpha_{z 0}>0\right)$. Eliminating $k_{x}$ from Eqs. (6)-(7) and multiplying both sides of the result by $h^{2}$ gives:

$$
\frac{\left(k_{z}^{\prime} h\right)^{2}}{R_{1}}+\frac{(\xi h)^{2}}{R_{2}}=1, \quad\left(\xi=\frac{\alpha_{z 0} \Delta}{\varepsilon_{z z}}\right),
$$

where $R_{I}$ and $R_{2}$ are defined respectively by:

$$
R_{1}=\frac{\Delta}{\varepsilon_{z z}^{2}}\left(\varepsilon_{z z}-1\right)\left(k_{0} h\right)^{2},
$$

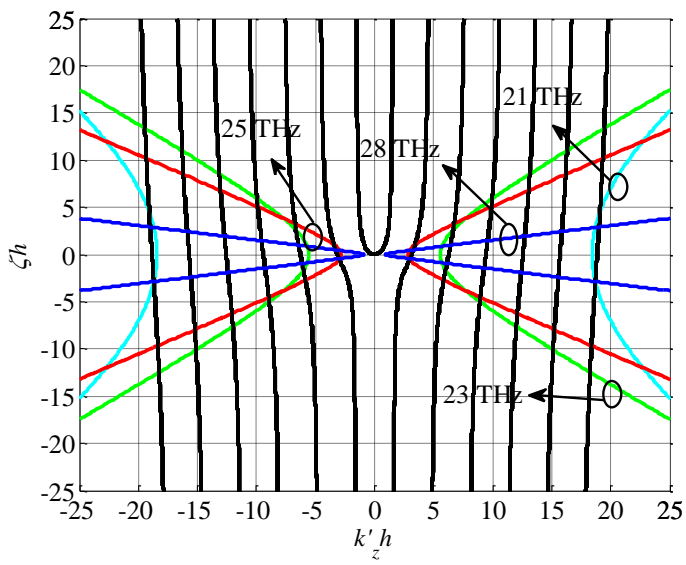

(a)

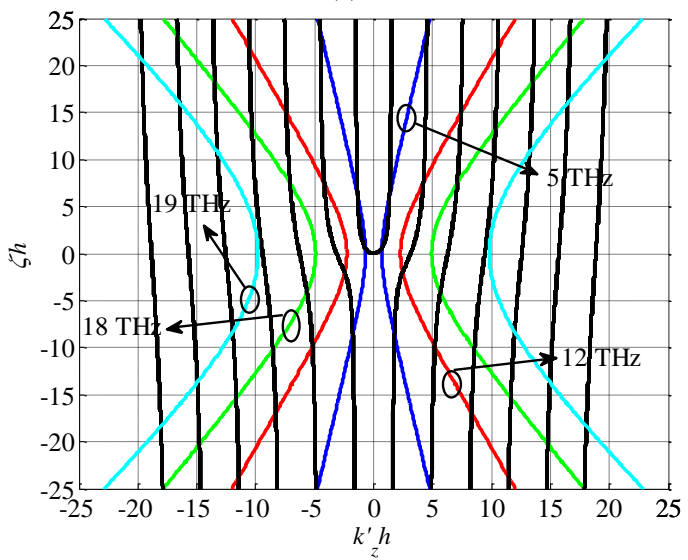

(b)

Fig. 2. Graphical solution of transcendental equations for TM wave mode of the grounded CNT slab with thickness $h=0.3 \lambda_{0}$ at frequencies below (a) and higher (b) than $f_{0}=20 \mathrm{THz}$. The considered slab is similar to [9] except loss which is removed.

$$
R_{2}=\frac{\Delta^{2}}{\varepsilon_{z z}^{2}}\left(\varepsilon_{z z}-1\right)\left(k_{0} h\right)^{2},
$$

which is the equation of a hyperbola in the $\xi h, k_{z}^{\prime} h$ plane (see Fig. 2). One can show that Eq. (5) can be rewritten as

$$
\xi h=k_{z}^{\prime} h \tan \left(k_{z}^{\prime} h\right),
$$

which is also plotted in Fig. 2. Equations (8) and (11) constitute a set of simultaneous transcendental equations which must be solved for the $\xi h$ and $k_{z}^{\prime} h$. A graphical representation of the solutions is shown in Fig. 2. The intersection of these curves implies a solution to both Eq. (8) and Eq. (11). We can see that $\xi h$ may be positive or negative for proper $\left(k_{z 0}=-\mathrm{j} \alpha_{z 0}\right)$ or improper $\left(k_{z 0}=\mathrm{j} \alpha_{z 0}\right)$ mode, respectively. Since the hyperbola intersects more than one branch of the tangent function, implying that more than one TM mode can propagate. If we know the 


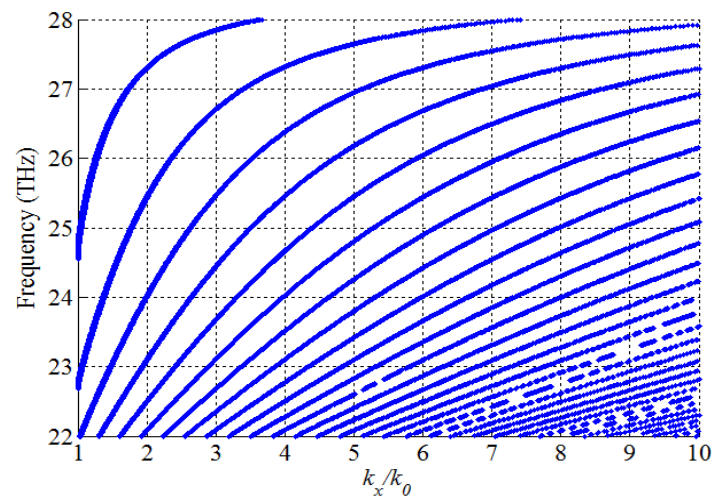

(a)

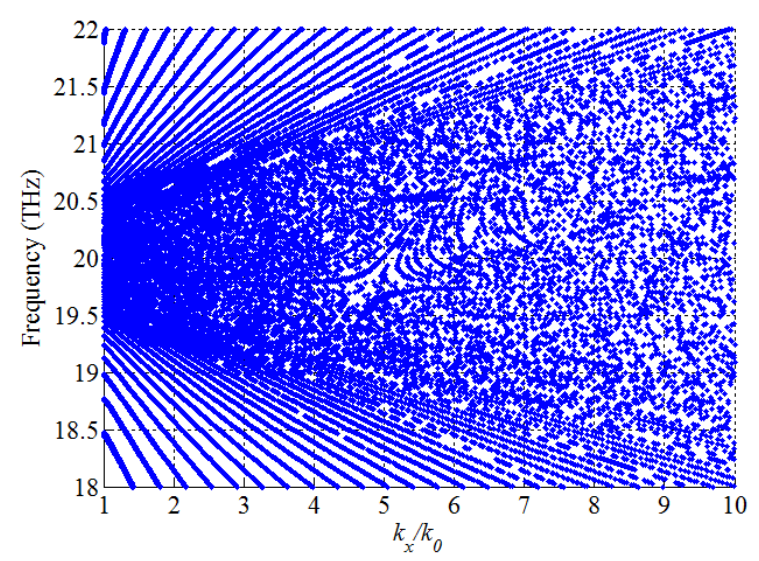

(b)

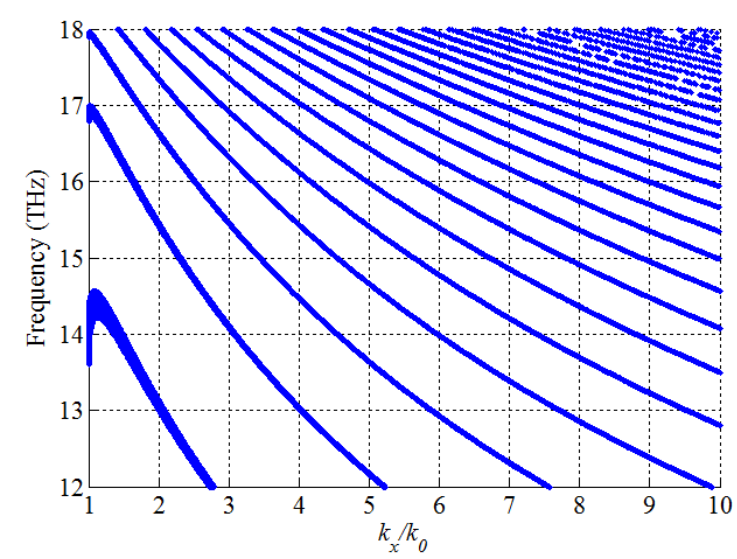

(c)

Fig. 3. Wave propagation constant for a grounded CNT slab with thickness $h=0.3 \lambda_{0}$ at frequencies below (a), near (b) and higher (c) than $f_{0}=20 \mathrm{THz}$. The considered slab is similar to [9] except loss which is removed. $\xi h$ and $k_{z} h$ from graphical solution then the $k_{x}$ can be found as:

$$
\frac{k_{x}}{k_{0}}=\frac{1+\frac{\varepsilon_{z z} \xi^{2}}{k_{z}^{\prime 2} \Delta}}{1+\frac{\xi^{2}}{k_{z}^{\prime 2} \Delta}} .
$$

The numerical solution of Eqs. (8) and (11) for TM modes is shown in Fig. 3. Each branch of the tangent function is one of TM modes (see Fig. 3(b)). The frequency $f_{0}$ is the limiting point for the cutoff frequencies of a countable spectrum of modes propagating in the HM slab. It is explained by a high value of the tangent argument $k_{z}^{\prime} h$, that causes rapid oscillations of this function in the vicinity of $f_{0}$. As can be seen from Fig. 2(b), if the frequency increases, the hyperbola curve shifts to such an extent that it does not intersect with some branches of the tangent curves, which means that we have a higher cutoff frequency for those branches (modes). We can also see this effect in Fig. 3(c), where modes propagate below $f_{0}$ and these modes are backward modes. Again, when the frequency is higher than $20 \mathrm{THz}$, the hyperbola curve shifts toward the centre and the forward TM modes have lower cut-off frequencies. We can see this effect in Fig. 3(a).

\section{References}

[1] D.R. Smith, D. Schurig, Phys. Rev. Lett. 90, 077405 (2003).

[2] P.A. Belov, et al., Phys. Rev. B 67, 113103 (2003).

[3] I.S. Nefedov, Phys. Rev. B 82, 155423 (2010).

[4] Z. Jakob, I. Smolyaniniv, E.E. Narimanov, Appl. Phys. Lett. 100, 181105 (2012).

[5] N. Poddubny, et al., Phys. Rev. B 86, 035148 (2012).

[6] V.N. Ivanov, et al., Izvestiya Vysshikh Uchebnikh Zavedenii Radiofizika (Radiophysics and Quantum Electronics) 32, 764 (1989).

[7] A. Yakovlev, et al., IEEE Trans. Microw. Theory Tech. 57, 2700 (2009).

[8] I. S. Nefedov, S.A. Tretyakov, Phys. Rev. B 84, 113410 (2011).

[9] I. S. Nefedov, C.R. Simovski, Phys. Rev. B 84, 195459 (2011).

[10] S.-A. Biehs, M. Tschikin, P. Ben-Abdallah, Phys. Rev. Lett. 109, 104301 (2012).

[11] Yu Guo, C. L. Cortes, S. Molesky, Z. Jacob, Appl. Phys. Lett. 101, 131106 (2012)

[12] S.-A. Biehs, M. Tschikin, R. Messina, P. Ben-Abdallah, Appl. Phys. Lett. 102, 131106 (2013).

[13] B. Liu, S. Shen, Phys. Rev. B 87, 115403 (2013).

[14] G.Y. Slepyan, S.A. Maksimenko, A. Lakhtakia, O. Yevtushenko, A.V. Gusakov, Phys. Rev. B 60, 17136 (1999).

[15] S. M. Hashemi, I. S. Nefedov, Phys. Rev. B 86195411 (2012). 